\title{
Detection of Bitter Taste Molecules Based on Odorant-Binding Protein-Modified Screen-Printed Electrodes
}

\author{
Zetao Chen, Qingqing Zhang, Jianzhen Shan, Yanli Lu,* and Qingjun Liu \\ Cite This: ACS Omega 2020, 5, 27536-27545 \\ Read Online
}

ABSTRACT: Bitter taste substances commonly represent a signal of toxicity. Fast and reliable detection of bitter molecules improves the safety of foods and beverages. Here, we report a biosensor using an easily accessible and cost-effective odorant-binding protein (OBP) of Drosophila melanogaster as a biosensitive material for the detection of bitter molecules. Based on the theoretical evaluation of the protein-ligand interaction, binding energies between the OBP and bitter molecules were calculated via molecular docking for the prediction and verification of binding affinities. Through one-step reduction, gold nanoparticles (AuNPs)

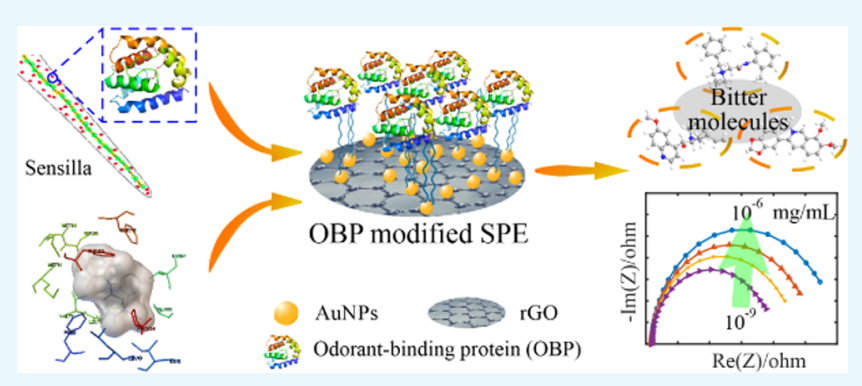
and reduced graphene oxide ( $\mathrm{GGO}$ ) were deposited on the screen-printed electrodes for improving the electrochemical properties of electrodes. After the electrodes were immobilized with OBPs via layer-by-layer self-assembly, typical bitter molecules, such as denatonium, quinine, and berberine, were investigated through electrochemical impedance spectroscopy. The bitter molecules showed significant binding properties to the OBP with linear response concentrations ranging from $10^{-9}$ to $10^{-6} \mathrm{mg} / \mathrm{mL}$. Therefore, the OBP-based biosensor offered powerful analytic techniques for the detection of bitter molecules and showed promising applications in the field of bitter taste evaluation.

\section{INTRODUCTION}

Bitter taste, as primary gustation, plays an important role in many fields, such as food safety and the pharmaceutical industry. ${ }^{1,2}$ Bitter taste substances are commonly assumed as signals for toxicity and prevent humans from ingestion of potentially spoiled food, which is important for the continuation of life. ${ }^{3}$ Moreover, the bitter taste of many clinical drugs indicates the possibility of drug repurposing; for example, the bitter taste of pediatric drugs can cause compliance and safety problems when given to the children., Thus, fast and reliable detection of bitter taste substances can improve the life experience and promote drug development. Recently, biosensors for the detection of bitter molecules are usually constructed based on gustatory tissue cells and bitter receptors. $^{5-7}$ However, the sensitive tissue cells that are extracted and cultivated with a complex process are highly dependent on the culture environments such as temperature and nutrition, making them impractical for applications in the biosensors. Due to high specificity, the bitter taste receptors such as T2R1 and T2R4 are the most desirable bitter-sensitive materials, which are usually produced from taste tissue cells and accessible for quantitative immobilization on electrodes to improve the repeatability. ${ }^{8,9}$ However, the difficulty in accessing bitter receptors led researchers to look for suitable alternative sensitive proteins for the detection of bitter molecules.

Odorant-binding proteins (OBPs), as major proteins existing in the nasal mucosa of vertebrates and the antennae of insects for the binding of odorant molecules, contribute to the recognition process of surrounding odorants in the olfactory sensation system, which usually participated in the construction of olfactory biosensors. ${ }^{10-12}$ Involving as ligand selector and transporter, the OBPs could bind the odorant molecules through the specific amino acid sites in the structural cavity and transport the ligands to the odorant receptors in the cell membrane. ${ }^{11,13}$ Triggering the pathway of $\mathrm{G}$ protein-coupled signals, the odorant receptors activate the olfactory neurons and cause the related olfactory behavioral response. Moreover, the isolation and purification processes of the OBPs are easier than those of the receptor proteins, which makes them easily accessible and even makes available their commercial products. ${ }^{14,15}$ As globular proteins of about 10-30 $\mathrm{kDa}$, the binding properties of OBPs are robust enough for withstanding different conditions, such as wide ranges of $\mathrm{pH}$ values and temperatures. ${ }^{11}$ Therefore, the OBPs are stable enough as promising sensitive materials for the construction of biosensors and will greatly enhance the practicability.

Received: August 24, 2020

Accepted: September 29, 2020

Published: October 15, 2020

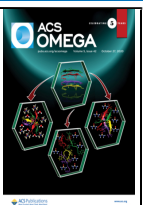




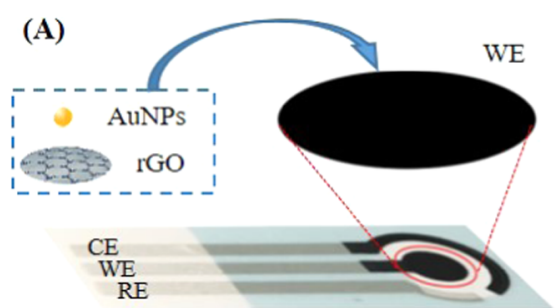

Reduction / Deposition

(B)

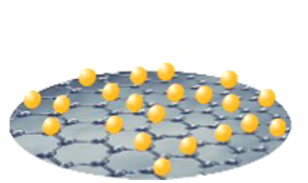

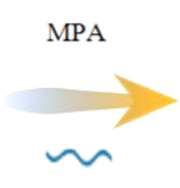

(D)

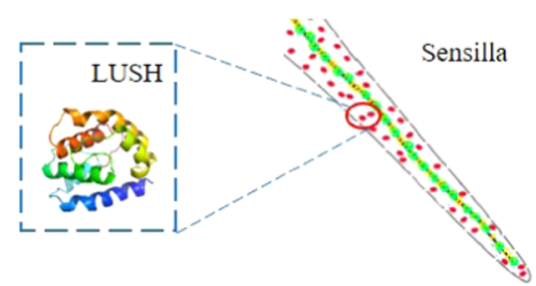

$\mathrm{EDC} / \mathrm{NHS}$

(C)

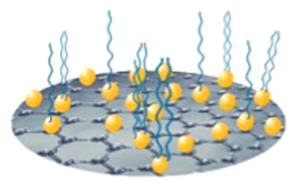

(E)

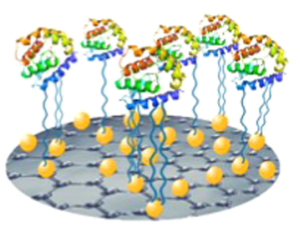

Figure 1. Schematic diagram of the OBP-based biosensor. (A, B) Gold nanoparticles and reduced graphene oxide were deposited on the screenprinted working electrodes through one-step electrochemical reduction. (C) MPA was immobilized on the gold nanoparticles through Au-S bonds. (D) OBP, termed as LUSH, was extracted from the sensilla of the fruit fly. (E) OBP was immobilized on MPA through peptide bonds.

Besides expression in olfactory tissues for odorant recognition, the OBPs were also expressed in other tissues such as gustatory sensilla, where they act as neuron activators, $^{16-19}$ whose physiological functions need to be studied further. As one of the OBP mutants, LUSH is generated in the process of mobilizing the $P$ element from the ET249 stock and widely expressed in pheromone-sensing neurons, which are mainly conserved among the antenna of fruit fly, Drosophila melanogaster. ${ }^{20}$ In the gustation system, OBPs function for the sensation activation of sweet by affecting the activity of gustatory receptor neurons. ${ }^{21}$ While in the presence of bitter molecules, the response to sweet taste was inhibited by the OBPs, which were occupied by attracting the bitter molecules and suppressing the activities of gustatory receptor neurons. ${ }^{16,21}$ The essential functions of OBPs in affecting the gustatory mechanism indicate that the OBPs could be combined with bitter ligands. ${ }^{16,17}$

In this study, we report a bitter biosensor based on the binding mechanisms of OBPs and bitter molecules, which uses the good performances of OBPs for the detection of bitter molecules. Based on the theoretical evaluation of the binding affinities of OBPs and bitter molecules, molecular docking was calculated and analyzed for explanations and verifications of the binding properties, through which the binding postures of bitter molecule ligands were predicted. With the gold nanoparticles and reduced graphene oxide (rGO)-modified screen-printed electrodes, an easily accessible and cost-effective OBP was immobilized for the detection of bitter molecules, such as denatonium, quinine, and berberine, via electrochemical impedance spectroscopy. This OBP-based bitter biosensor could be used for detecting trace levels of bitter substances and is promising for applications in bitter taste evaluation.

\section{EXPERIMENTAL SECTION}

2.1. Recombinant OBPs and Reagents. As an OBP, LUSH was extracted from the antenna of fruit fly, $D$. melanogaster, for the construction of the bitter biosensor based on the combination of OBP and bitter molecules. The expression and purification processes of the active recombinant
OBP are completed at MyBiosource, Inc. The target DNA was cloned from cDNA by plasmid rescue. ${ }^{22}$ The appendage cDNA was prepared from mRNA using isolated appendages. ${ }^{23}$ Therefore, a DNA sequence encoding the LUSH protein with a His- $\mathrm{B}_{2} \mathrm{M}$ tag at the $\mathrm{N}$-terminal and a Myc tag at the Cterminal in the expression host Escherichia coli. The recombinant OBP consisted of 124 amino acids with a molecular mass of $19 \mathrm{kDa}$ was then prepared. This target OBP was suspended at $0.1 \mathrm{mg} / \mathrm{mL}$ in a storage buffer solution (prepared with $0.2 \mu \mathrm{m}$ filtered, sterile $10 \mathrm{mM}$ Tris- $\mathrm{HCl}, 1$ $\mathrm{mM}$ EDTA, and $50 \%$ glycerol, $\mathrm{pH}=8.0$ ) for the subsequent experiments.

Graphene oxide (XFNANO, China) was utilized to form reduced graphene oxide ( $\mathrm{rGO})$ for the modification of electrodes. Chloroauric acid $\left(\mathrm{HAuCl}_{4}\right)$ was prepared for the synthesis of gold nanoparticles (AuNPs). N-Hydroxysuccinimide (NHS) and 1-ethyl-3-(3-dimethylaminopropyl) carbodiimide (EDC) dissolved in 2-(4-morpholino)ethanesulfonic acid (MES) were used for the immobilization of the OBP. Denatonium, quinine, and berberine were prepared at $10^{-9}$, $10^{-8}, 10^{-7}$, and $10^{-6} \mathrm{mg} / \mathrm{mL}$ with ultrapure water for the measurements. Acetic acid $(\mathrm{pH}=5)$ and $\mathrm{HCl}(\mathrm{pH}=6)$ were prepared as sour taste, sodium glutamate was prepared as umami taste, sodium chloride $(\mathrm{NaCl})$ was prepared as saline taste, and sucrose was prepared as sweet taste samples. Ferricyanide/ferrocyanide $\left(\mathrm{Fe}(\mathrm{CN})_{6}{ }^{3-/ 4-}\right)$ prepared at $10 \mathrm{mM}$ in $0.1 \mathrm{M}$ potassium chloride $(\mathrm{KCl})$ was treated as a redox couple. The cross-linker termed 3-mercaptopropionic acid (MPA) containing a sulfhydryl and a carboxyl on each end was prepared at $50 \mathrm{mM}$ for the immobilization procedure of OBP. All of the chemical reagents were of analytical grade and purchased from Sigma-Aldrich.

2.2. Theoretical Evaluation of OBP Binding to Bitter Molecules. To evaluate the binding property between the OBP and bitter molecules, a three-dimensional (3D) structure of the OBP was modeled through SWISS-MODEL via the ExPASy web server. ${ }^{24}$ The binding postures between the OBP and various bitter molecules were predicted by AutoDock (free and available under the GNU General Public License). ${ }^{25}$ The three-dimensional structures of denatonium, quinine, berber- 


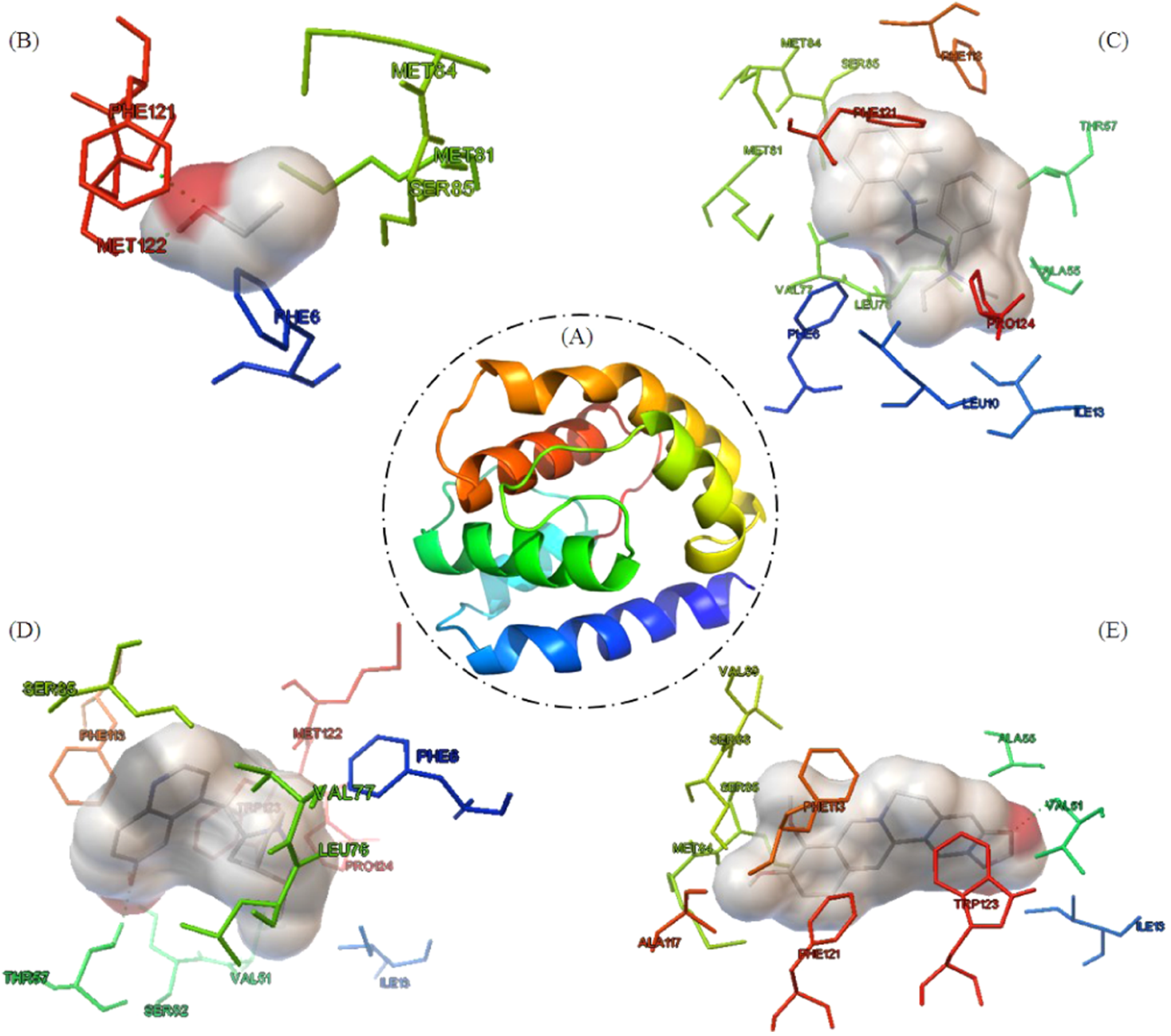

Figure 2. Molecular docking of ligands binding to the OBP named LUSH. (A) Three-dimension structure of the OBP, through which six helices could be clearly seen. Docking poses of ethanol (B), denatonium (C), quinine (D), and berberine (E) with the OBP were calculated, through which the binding cavities were marked as gray and encapsulated the related ligands (related amino acid residues were labeled).

ine, and ethanol were obtained from the PubChem Compound Database of the National Center for Biotechnology Information. A genetic algorithm was used as the search algorithm for the molecular docking process, while an integrated scoring function was employed to evaluate the docking results. Before docking, the OBP molecule was preprocessed with water deletion, polar hydrogen addition, and Kollman charge addition. With the analysis of molecular docking, the top 10 docking postures ranked by the corresponding binding scores were preserved to find the most probable binding modes.

2.3. Fabrication of Screen-Printed Electrodes. The screen-printed electrodes fabricated by volume screen printing were used as electrochemical electrodes for the detection of bitter molecules to decrease the difference between electrodes. Polyethyleneglycol terephthalate, which had been proved as a waterproof material, was used as substrates of the electrodes. The size of the electrodes was designed in $30 \mathrm{~mm} \times 8 \mathrm{~mm} \times$ $0.2 \mathrm{~mm}$ to satisfy the miniaturization. Conductive graphite paste with high stability was used for the printing of working electrodes (circles with $4 \mathrm{~mm}$ in diameter) and counter electrodes. The reference electrodes were printed using silver/ silver chloride paste through a mask. Silver paste was used for the printing of wires with high conductivity. At the end of the wires near the working electrodes, an insulation tape was pasted on the surface of the substrate to isolate wires and electrodes. For connecting screen-printed electrodes to the electrochemical impedance spectroscopy detection system, a self-designed connection socket was used.

2.4. Modification of the Electrochemical Electrodes. Modification of the screen-printed electrodes with rGO and AuNPs through one-step reduction can increase the conductivity and enlarge the specific surface area of the electrodes. ${ }^{26}$ After thoroughly rinsing with ultrapure water, successive cyclic voltammetry $(\mathrm{CV})$ sweeps were performed as preprocessing until a steady curve appears, making the electrode stable. A $1 \mathrm{mg} / \mathrm{mL}$ GO suspension in $0.5 \mathrm{M}$ $\mathrm{Na}_{2} \mathrm{SO}_{4}$ was prepared through the ultrasonic dispersion. Afterward, $\mathrm{HAuCl}_{4}$ aqueous solution with a mass ratio of $0.5 \%$ was mixed with the $\mathrm{GO}$ suspension at a volume ratio of 30:1 $\left(\mathrm{GO} / \mathrm{HAuCl}_{4}\right)$. The mixed solution was then dropped on the cleaned working electrodes (Figure 1A). Through electrochemical reduction, AuNPs and rGO were deposited on the electrodes simultaneously (Figure 1B). After the modified electrodes were rinsed with ultrapure water, the electrodes were immersed in a $50 \mathrm{mM}$ MPA solution for $24 \mathrm{~h}$ to link the MPA on AuNPs via $\mathrm{Au}-\mathrm{S}$ bonds while leaving the carboxyl of MPA free for the immobilization of the OBP (Figure 1C). 

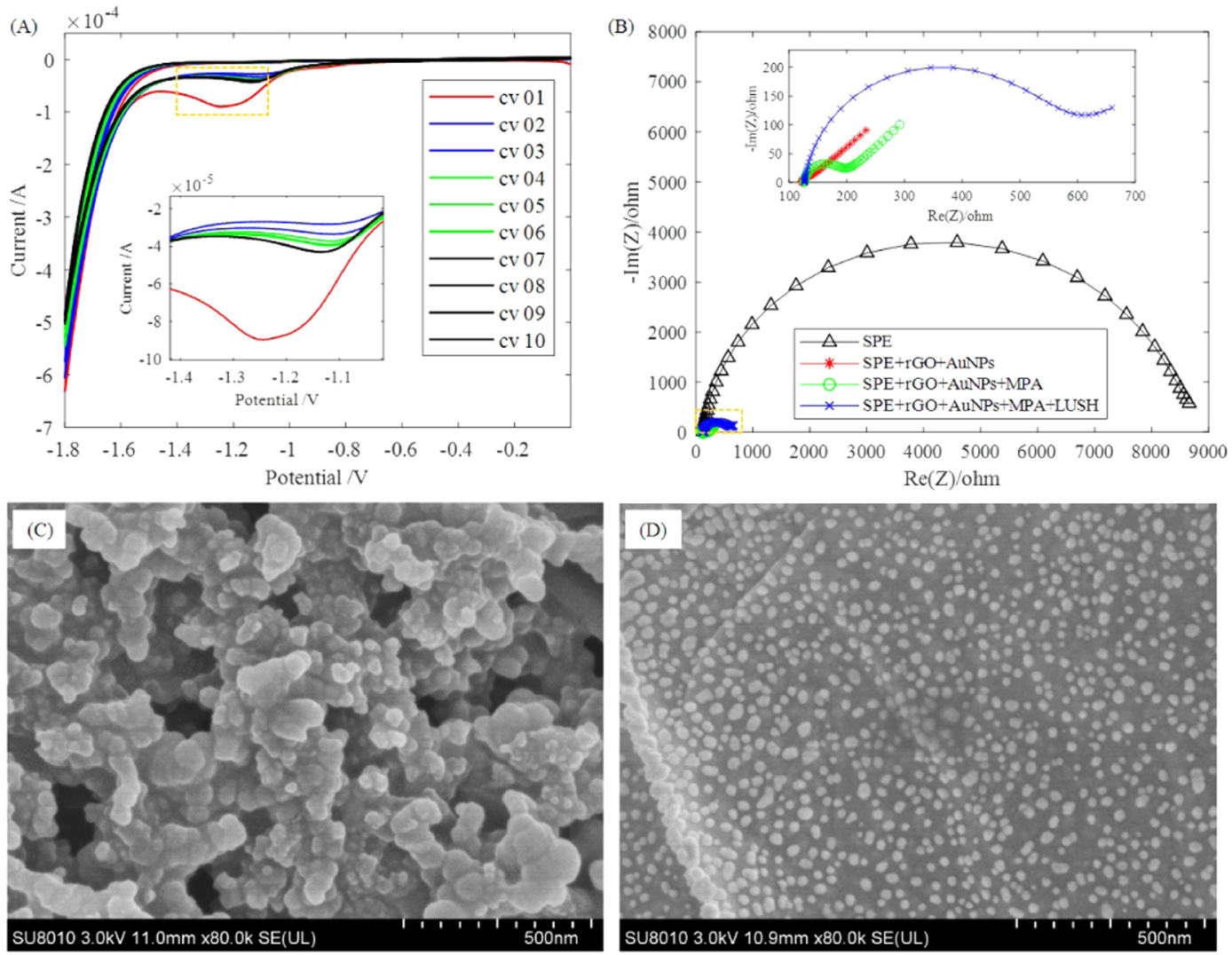

Figure 3. Reduction and characterization of the gold nanoparticles and reduced graphene oxide on the screen-printed electrodes. (A) Cyclic voltammograms of the electrochemical reduction of graphene oxide to reduced graphene oxide and $\mathrm{HAuCl}_{4}$ to gold nanoparticles (the inset shows the close-up of curve peaks). (B) Characterization of electrochemical impedance spectroscopy for each modification step. (C) Scanning electron microscopy (SEM) image of the bare screen-printed working electrode. (D) SEM image of the screen-printed working electrode modified by reduced graphene oxide and gold nanoparticles.

Through the cross-linker MPA, the OBP was immobilized onto the screen-printed electrodes via layer-by-layer selfassembly. The OBP is a protein extracted from the sensilla of fruit fly containing at least one amidogen at the end of the amino acid chain (Figure 1D). A mixed solution of $2 \mathrm{mM}$ EDC and $5 \mathrm{mM}$ NHS in a volume ratio of 1:1 was dropped on the working electrode to activate the carboxyl groups of the MPA linked on the AuNPs. After the $\mathrm{pH}$ of the mixed solution was adjusted to $7.4,30 \mu \mathrm{L}$ of $0.1 \mathrm{mg} / \mathrm{mL}$ OBP was added to the electrodes. Under the assistance of this EDC/NHS, the amidogen end of the OBP protein was attached to the carboxyl end of MPA through dehydration synthesis. Dehydration synthesis was performed under $4{ }^{\circ} \mathrm{C}$ for $2 \mathrm{~h}$ to form amide linkages. Afterward, the electrodes were rinsed with $\mathrm{PBS}$ buffer solution to remove residual reagents including EDC/NHS and unbonded OBP. Finally, the electrodes were successfully immobilized with OBP and stored under $4{ }^{\circ} \mathrm{C}$ for subsequent experiments (Figure 1E).

2.5. Bitter Taste Molecule Sensing with Electrochemical Impedance Spectroscopy. Electrochemical impedance spectroscopy was used for the measurements of bitter taste molecules. Denatonium, quinine, and berberine were detected as typical bitter molecules, while the other basic tastes were tested to explore the specificity; for example, acetic acid and $\mathrm{HCl}$ were tested as sour, sodium glutamate was tested as umami, $\mathrm{NaCl}$ was tested as saline, and sucrose was tested as sweet molecules. All of the taste molecules were tested under the same conditions. During the experiments, taste molecules at different concentrations were added to the screen-printed electrodes containing redox couples. The $\mathrm{pH}$ of analytes except sour ones was adjusted to 7 to exclude the influences of $\mathrm{pH}$ on the electrodes. After each measurement, the tested solution was removed to clean the residual taste molecules and the electrodes were then immersed in ultrapure water for $2 \mathrm{~min}$ for the desorption of ligands to recover the immobilized OBP for the next detection cycle. Parallel experiments with the same screen-printed electrode for each taste substance were performed four times to exclude the contingency of the detection. Parallel experiments with different screen-printed electrodes for each taste substance were performed four times to verify the stability of the electrodes. In the electrochemical measurement, drifts of the sensor's responses affect the detection results greatly. To eliminate response drifts, a debaseline method was utilized. Before each test of analytes, a test with the basal solution was performed as a control experiment. Then, the detection results were obtained by subtracting the impedance of the basal solution from the impedance of the analyte.

\section{RESULTS}

3.1. Binding Affinities of OBPs and Bitter Taste Molecules. Computational biology plays significant roles in the field of molecular structure prediction and protein-protein interaction, which enables new approaches to probe biological function. Molecular docking, as a branch of computational biology, was utilized to predict and evaluate the interactions 

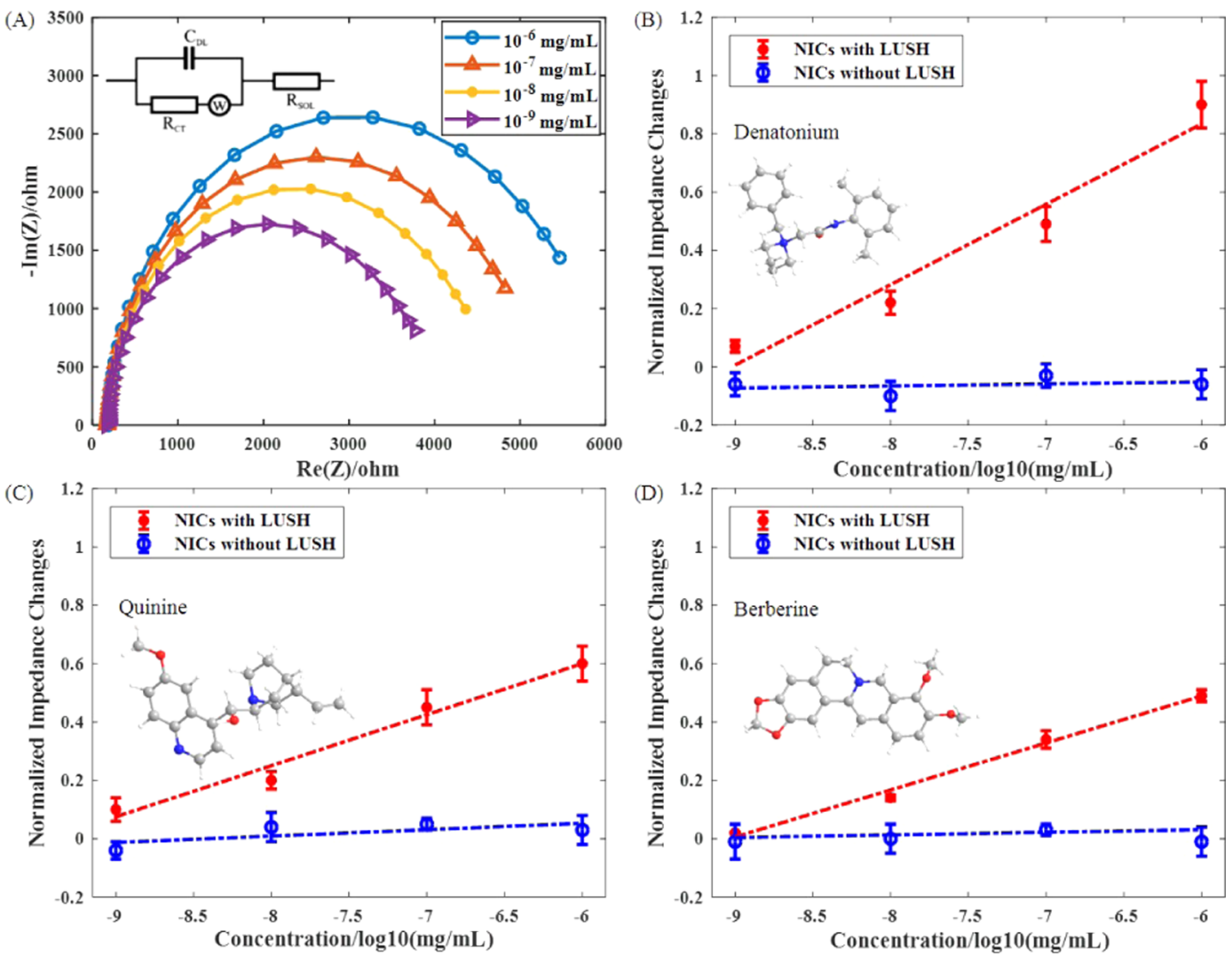

Figure 4. Detection of bitter taste molecules with the OBP-based biosensor. (A) Nyquist plots for the detection of denatonium at concentrations of $10^{-9}, 10^{-8}, 10^{-7}$, and $10^{-6} \mathrm{mg} / \mathrm{mL}$ (the inset shows the equivalent circuit consisting of four elements: $R_{\mathrm{SOL}}$, the solution resistance; $C_{\mathrm{DL}}$, the constant phase element; $W$, the Warburg impedance; $R_{\mathrm{CT}}$, the charge-transfer resistance). The normalized impedance changes (NICs) along with different concentrations of denatonium (B), quinine (C), and berberine (D) were plotted and fitted through the least-squares method $(n=4)$. Error bars for each NIC indicate the standard deviation, and the insets show the corresponding $3 \mathrm{D}$ molecular structures.

between the OBPs and their related ligands. The threedimensional structure of the OBP predicted through homology modeling contains six $\alpha$-helices (Figure 2A). These helices formed a cavity that can stably bind the relative ligands. The binding properties and binding modes of the OBP with ethanol (pheromone of the OBP) molecules (Figure 2B) and bitter taste molecules such as denatonium (Figure 2C), quinine (Figure 2D), and berberine (Figure 2E) were evaluated through AutoDock, illustrating that the amino acid residues of the OBP contribute most to the binding affinities. Hydrogen bonds were represented as dashed green lines, which crossed the red area of the gray cavity. Residues in the inner cavity contributing to the force field were also labeled and colored based on their located helices. According to molecular docking, serine (SER) formed electrostatic forces as it is polar, methionine (MET) contributed to the force of hydrogen bonds, and the benzene ring of phenylalanine (PHE) formed $\pi-\pi$ bonds. These three residues are important in the cavity of the OBP for molecular docking, as ethanol formed hydrogen bonds with MET and PHE and quinine, denatonium, and berberine formed $\pi-\pi$ bonds with PHE through the benzene rings. Based on the interactions between OBP and ligands, the binding postures were predicted and restricted by the helices formed in the cavity of the OBP (Figure S1).

The force field of protein-ligand binding was calculated through the scoring functions, indicating the binding properties of the OBP and bitter molecules. The scoring function consisting of dispersion/repulsion, hydrogen bonds $(\mathrm{H}$ bonds), electrostatics, and desolvation was utilized to evaluate the molecular docking affinities, where a larger negative score means stronger binding forces. Based on the scoring function, detailed molecular docking scores were analyzed to evaluate the contribution factors (Table S1). The total internal in the table represents dispersion/repulsion, which were interactions between carbon-carbon atoms. The van der Waals (vdW) Hbond desolvation energy contributes to the binding energy based on the vdW potentials, H-bond potentials, and the desolvation potentials. The vdW potentials were assigned for electrostatics that reflected the interactions of oppositely charged atoms, the $\mathrm{H}$-bond potentials were forces of oxygenhydrogen interactions, and the desolvation potentials were interactions between the surrounded atom (usually carbon atom) and the sheltered solvent. Based on molecular docking, combined with the intermol energy that was related to the ligands' molecular weight, the bitter molecules showed a significant binding affinity to the OBP.

3.2. Characterization of the OBP-Modified Electrodes. Through one-step electrochemical reduction, the screenprinted electrodes were deposited with rGO and AuNPs. With the modification of $\mathrm{rGO}$, the conductivity of the electrodes was improved. The AuNPs were deposited not only for enlarging the specific surface area but also for forming $\mathrm{Au}-\mathrm{S}$ bonds for the immobilization of the OBP. Based on the scanning of $\mathrm{CV}$ at different rates, the cyclic voltammogram at a scanning rate of $50 \mathrm{mV} / \mathrm{s}$ was relatively stable and was selected for the subsequent characterization procedures (Figure S2). 
While in the electrochemical reduction process, after sweeping 10 times, the CV curves trend to be stable and the last few rounds overlapped, indicating that reduction and deposition were completed (Figure 3A). As many hydroxy and carboxyl groups were enriched on graphene oxide, the graphene oxide possessed unique physical and chemical properties, but these groups could be also easily reduced. An obvious large peak of the reduction $\mathrm{CV}$ curve appeared at about $-1.25 \mathrm{~V}$ in the first cycle, starting at a potential of $-1.0 \mathrm{~V}$, which could be attributed to the reduction of functional groups like hydroxy and carboxyl of the graphene oxide. After several reduction rounds, the remained groups were completely reduced. During the reduction period, no oxidation peaks were found, indicating that the reduction of graphene oxide is irreversible and rGO is relatively stable for the detection.

After each modification step, electrochemical impedance spectroscopy was performed to characterize the surface properties of the screen-printed working electrodes (Figure $3 B)$. From the Nyquist plots, the impedance of the electrodes was decreased compared to that before the reduction, which means that the conductivity of the electrodes was increased. After the cross-linker MPA was linked to the working electrodes through $\mathrm{Au}-\mathrm{S}$ bonds, the impedance was slightly increased because the small MPA reduced the exchange area for electrons. With the immobilization of the OBPs, the impedance of the screen-printed electrode was increased, which was caused by the restriction of electron passage via OBP. The characterization for each modification step through $\mathrm{CV}$ was consistent with the characterization by electrochemical impedance spectroscopy (Figure S3). Moreover, through morphological characterization by scanning electron microscopy (SEM, Philips XL-30 ESEM), roughs and holes can be observed on the working electrodes before the modification of nanomaterials (Figure 3C). After reduction, rGO and AuNPs were modified on the surface of working electrodes (Figure 3D). The sphere structures and several wrinkles on the surface indicated the success of AuNP deposition and rGO reduction. Furthermore, the electrochemical reduction of $\mathrm{HAuCl}_{4}$ with lower concentrations was not enough for AuNP modification; however, the reduction with higher concentrations could cause agglomeration of AuNPs (Figure S4). Thus, when the concentration of $\mathrm{HAuCl}_{4}$ was $0.5 \%$, the modified AuNPs were evenly distributed with relatively uniform size and without large agglomeration.

3.3. Detection of Bitter Taste Molecules with the OBP-Based Biosensor. Based on the modified electrodes, the OBP-based biosensor was constructed for the detection of bitter molecules. Electrochemical impedance spectroscopy, which was often utilized to explore the kinetics of the electrode process, could be analyzed by an equivalent circuit (inset of Figure 4A). Elements of this equivalent circuit, such as the interfacial charge-transfer resistance $\left(R_{\mathrm{CT}}\right)$ of the electrodes, were measured for the evaluation of bitter taste. Therefore, the $R_{\mathrm{CT}}$ of the Nyquist plots was chosen as the sensing parameter for monitoring protein-ligand interactions. As one of the most bitter-tasting substances known, denatonium was detected to explore the specific bindings between the bitter molecules and the OBP. For the detection of denatonium molecules, the impedance of electrodes increases with the increase of denatonium concentration, indicating that there are interactions between the OBP and denatonium (Figure 4A). As the normalized impedance changes (NICs) were calculated via the relative changes of $R_{\mathrm{CT}}$, based on the detection of denatonium,
NICs increased along with the denatonium concentration (Figure 4B). According to the fitting line, significant binding affinities between denatonium and the OBP were observed, which revealed that a concentration range from $10^{-9}$ to $10^{-6}$ $\mathrm{mg} / \mathrm{mL}$ was the linear working range of the OBP-based biosensor for denatonium. Moreover, NIC responses to the interactions between denatonium and electrodes without the immobilization of OBP showed no significant changes along with increasing denatonium concentration. Therefore, the bitter molecule of denatonium was sensitively detected by the OBP-based biosensor.

Through the electrochemical impedance spectroscopy measurements of bitter molecules such as denatonium, quinine, and berberine, the interactions between bitter molecules and the OBP were analyzed to investigate the sensitivity of the OBP-based biosensor for the detection of bitter taste molecules. Through the least-squares method, the detection of denatonium could be fitted as NICs $=0.276 \times$ $\log _{10} C+2.49(R$-square $=0.9575)($ Figure $4 \mathrm{~B})$, the detection of quinine could be fitted as NICs $=0.175 \times \log _{10} C+1.65(R$ square $=0.9761)$ (Figure $4 \mathrm{C})$, and the detection of berberine could be fitted as NICs $=0.161 \times \log _{10} C+1.455(R$-square $=$ 0.9918) (Figure 4D), where $C$ represents the concentration. According to these fitting functions, it could be observed that the binding affinity of denatonium is larger than that of quinine, while the binding affinity of quinine is larger than that of berberine. Slopes of these functions reflect the impedance of the screen-printed electrodes. A bigger slope means a larger barrier for the electron transfer, which can be used to classify different bitter molecules. Moreover, these three bitter taste molecules were also measured by electrodes without the immobilization of the OBP. NICs of the detections showed no significant difference along with the bitter molecule concentrations, which indicated that the NICs to bitter molecules detected by the OBP-based biosensor were caused by the interactions between bitter molecules and the OBP. As the experiments were consistent with the results of computational simulations, the OBP-based biosensors were helpful for confirming the binding properties of proteins with different ligands. Therefore, the OBP-functionalized biosensor provided a potential approach for detecting different bitter molecules.

The OBP-based biosensor had high stability and repeatability. Electrodes used in this sensor were screen-printed electrodes, of which the working electrode was printed by carbon paste. Carbon is a very stable element, which can greatly improve the stability of the sensor when used as a working electrode in a three-electrode system. For the modification of the electrode with AuNPs and rGO, a onestep reduction method was used, which greatly reduced the complexity of the modification process. During the modification, the amplitude of the reduction potential reached 1.2 V. However, for analyte detection using electrochemical impedance spectroscopy, the amplitude was set as $0.01 \mathrm{~V}$, which was much smaller than the reduction potential peak, meaning ignorable influences on the electrode surface. The $\mathrm{Au}-\mathrm{S}$ bond that links MPA to AuNPs on the electrode is a strong coordination bond. Under the assistance of EDC/NHS, the amidogen end of the OBP protein was attached to the carboxyl end of MPA through dehydration synthesis, the bond of which was stable enough for molecular detection. To improve the repeatability of this biosensor, the initial potential for electrochemical impedance spectroscopy was set as the open-circuit potential of the electrochemical workstation 
before each test to minimize the variations. For each detection, we repeated the process four times to ensure repeatability.

3.4. Detection of Basic Taste Substances by the OBPBased Biosensor. Basic tastes such as sour, sweet, salty, and umami were detected to explore the selectivity of this OBPbased biosensor (Figure 5). Sour tastes generate by responding

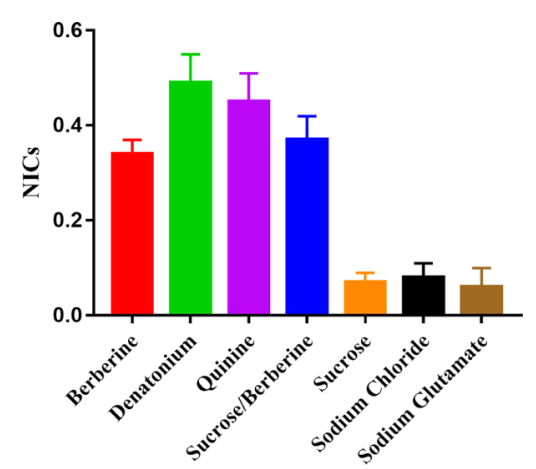

Figure 5. Comparison of the normalized impedance changes (NICs) of the OBP-based biosensors to different taste substances of berberine, denatonium, quinine, sucrose, sodium chloride, sodium glutamate, and the mixture of sucrose and berberine $(n=4)$, in which the bitter taste substances had significant electrochemical responding signals.

to acids through the mechanisms of interactions between taste receptor cells and hydrogen ions. Therefore, acetic acid was detected as an organic acid and the $\mathrm{HCl}$ was detected as an inorganic acid for sour taste by the OBP-based biosensor. Besides, sucrose was detected as sweet, $\mathrm{NaCl}$ was detected as salty, and sodium glutamate was detected as umami. The $\mathrm{pH}$ of sour taste was adjusted to 5 for comparative measurements. Under the condition of $\mathrm{H}^{+}$, the OBP immobilized on the electrodes could be released by the deconstruction of peptide bonds between MPA and OBP, which could increase the conductivity of the electrodes. The $\mathrm{H}^{+}$in sour solution led to the decrease of charge-transfer resistance and caused a below zero NIC response to sour taste. Therefore, the detection of sour substances by this OBP-based biosensor was not comparable.

Different concentrations of other taste substances were measured under the same conditions as for the detection of bitter molecules. For each taste substance, a concentration of $10^{-7} \mathrm{mg} / \mathrm{mL}$ was chosen for the analysis of selectivity. The NICs to sweet, salty, and umami were less than 0.1 , indicating that the binding of these taste molecules to the OBP was extremely week or there was even no binding between them. A mixed solution of sucrose and berberine was tested to explore the influence of sweet on bitter. For the increasing viscosity of the mixed solution caused by the addition of sucrose, the transfer of charger carriers in the solution decelerated, which decreased the NICs of the mixed solution. Therefore, the NICs to sweet and bitter substance mixed solution were larger than those of bare berberine under the same conditions. Thus, the OBP-based biosensor can be applied for distinguishing different taste substances owing to its high selectivity.

\section{DISCUSSION}

4.1. Roles of OBPs in Gustatory Detection. In the gustatory system, inhibitions exist among tastes. ${ }^{27}$ For the response of tastes, such as sweet, the interaction of sweet receptors and sweet molecules causes changes in the transmembrane domains, which proposed that the sweet tastes are induced by the penetration of sweet molecules into the nonpolar region of the lipid layer of the gustatory receptor membrane. ${ }^{28}$ Sweet receptors such as T1R3 are imbedded in the gustatory cell membrane for interaction with sweet taste molecules. Thus, the binding of sweet molecules with sweet taste receptors causes sweet taste responses. Generally, OBPs are expressed in the olfactory tissue cells for the recognition of odorants. ${ }^{15}$ However, OBPs also exist in the gustatory system and participate in the gustatory process. ${ }^{29,30}$ Electrophysiological and calcium imaging studies in D. melanogaster revealed that bitter molecules inhibit the activity of sweet sensory neurons, suggesting that the bitter-sweet integration occurred at the periphery. ${ }^{21,31}$ An OBP expressed in accessory cells in gustatory sensilla was found to be required for the inhibition of the neuronal activity of sweet in the presence of bitter molecules, ${ }^{16}$ revealing that the sweet-responsive neurons were inhibited and the response to sweet tastes was blocked through the OBPs. Therefore, the bitter molecules interact with the OBPs and affect sweet recognition. During the interaction of bitter molecules and OBPs, the OBPs bind with bitter molecules and work as transporters for bitter molecules. ${ }^{16,32,33}$ Based on the roles of OBP in gustatory sensilla, other OBPs such as OBP49a may also be available for bitter taste detection. $^{30,34}$

4.2. Binding Properties of OBPs and Bitter Taste Molecules. Based on the biological conformation of bitter substances and OBPs, ${ }^{35,36}$ the structure of bitter molecules affects the binding affinity a lot. The molecular structure of the benzene ring not only contributes to the generation of the bitter taste but also forms $\pi-\pi$ bonds for the affinity between molecules. ${ }^{37,38}$ Bitter taste molecules with different benzenebased structures were detected in this study, such as denatonium consisting of benzene and no nitrogen-containing heterocyclic ring (inset of Figure 4B), quinine consisting of benzene and one nitrogen-containing heterocyclic ring (inset of Figure 4C), and berberine consisting of benzene and more than one nitrogen-containing heterocyclic ring (inset of Figure $4 \mathrm{D})$. The benzene ring due to its molecular structure formed $\pi-\pi$ bonds that contributed to the binding affinity and achieved a balance; however, the nitrogen-containing heterocyclic ring broke the balance and weakened the binding energies. $^{3,39}$ Therefore, the total internal energy of denatonium containing benzene with no nitrogen-containing heterocyclic ring was higher than those of quinine and berberine; the total internal energy of berberine with more than one nitrogen-containing heterocyclic ring was weaker than that of quinine (denatonium, -1.22 ; quinine, -0.88 ; berberine, -0.21$)$. Although alcohol has no benzene rings, it can bind with the OBP mainly through hydrogen bonds.

Bitter taste receptors that combine with bitter taste molecules form a cavity for containing bitter molecules. ${ }^{40,41}$ Similarly, the OBP has six $\alpha$-helices, forming a cavity, that can bind with related ligands through the amino acid residuebinding sites. Through molecular docking, three residues positions of MET, SER, and PHE of the OBP were predicted and formed amino acid patterns, which contributed to the hydrogen bonds, $\pi-\pi$ bonds, and electrostatic forces that affect the binding affinities. The OBP named as LUSH, a six-helix olfactory sensitive protein, has similar binding mechanisms to bitter taste receptors. Therefore, the homology structure of the OBP with bitter taste receptors offered a promising opportunity to OBP for the detection of bitter molecules. 
Moreover, the OBPs are easily accessible, cost-effective, and highly stable to temperatures and solvents, ${ }^{13}$ indicating that the OBP-based biosensor can provide a promising platform for the detection of bitter taste molecules in various situations.

4.3. OBP-Based Biosensors for the Detection of Bitter Taste Substances. Our results confirmed that the biosensor developed using the odorant-binding protein as a biosensitive material for the detection of bitter molecules had high sensitivity and selectively. The variations between one sensor and another are still issues to be resolved. In our work, screenprinted electrodes were treated as electrochemical electrodes, which were fabricated by volume screen printing. The fabrication in batch can minimize the difference between electrodes. As the electrochemical properties of the screenprinted electrodes were highly dependent on the number of AuNPs and the coverage of rGO, the one-step reduction method was utilized to reduce the complexity of the modification and maintain maximum consistency among electrodes. The same preprocessing of successive cyclic voltammetry sweeps was performed to make the electrodes stable. All of the electrodes were treated with the same parameters. Although the differences between electrodes cannot be completely excluded, the sensor to sensor variations can be minimized through these batch fabrication and modification processes. As a two-dimensional nanomaterial with high conductivity, rGO is commonly used for the modification of electrodes to improve the electrical properties of electron transfer. ${ }^{42}$ The nanomaterial AuNPs are used often to modify the electrodes for increasing the specific area to enlarge the binding area. ${ }^{43}$ Based on the rGO- and AuNPmodified screen-printed electrodes, the stability and sensitivity of the biosensor were improved. Therefore, based on the enhanced modification of the electrodes, electron transfer of the electrodes evaluated for detecting bitter taste molecules was of high stability and sensitivity. As a zero-length crosslinker, the MPA contained a sulfhydryl and a carboxyl group on each end. The sulfhydryl end binds to the AuNPs through a $\mathrm{Au}-\mathrm{S}$ bond and the carboxyl end binds to the OBP via a peptide bond formed by the dehydration condensation reaction, both linkers of which are stable for electrochemical measurements. ${ }^{44}$ Based on the ignorable length of MPA, the influence of cross-linkers on detection was negligible, which contributed a lot to the high sensitivity of the OBP-based biosensor.

Compared with bitter biosensors developed using organ tissues, ${ }^{45,46}$ cells, ${ }^{47-49}$ bitter taste receptors, ${ }^{9}$ and other materials $^{50,51}$ as sensitive elements, the OBP-based biosensor showed advantages among them (Table 1). Bitter-sensitive tissue cells like the intact taste epithelium of rat can visibly detect the changes of bitter substances. The tissue can also provide three-dimensional formation with inherent interactions between neighboring cells. ${ }^{47}$ However, despite their high specificity, the impracticality of tissue cell cultures limited the application of tissue cell-based bitter biosensors. Due to the high specificity of bitter receptors to bitter molecules, the bitter receptors such as T2R4 were usually used for the detection of denatonium using a quartz crystal microbalance. ${ }^{9}$ With a low detection limit of $1.58 \mu \mathrm{M}$, this bitter receptor-based biosensor showed good performance for the detection of bitter taste. However, as a $\mathrm{G}$ protein-coupled receptor, the bitter receptor has seven transmembrane $\alpha$ helixes, which showed difficulty in the isolation and purification of bitter receptors. ${ }^{52}$ Thus, the OBPs are excellent alternatives for the construction of bitter




biosensors. Besides, the limits of detection using the OBPbased biosensor are even lower than those using bitter taste receptor-based biosensors. Therefore, through the OBP-based biosensor, bitter taste molecules could be detected with low concentrations. Different from the detection of standard reagents, the detection of actual samples is affected by various external environmental factors, which will also affect the detection accuracy of the sensor. On the other hand, not all of the bitter molecules could be detected by this OBP-based biosensor. Nevertheless, these limitations should be solved in future research.

\section{CONCLUSIONS}

In this study, based on the binding mechanisms between bitter molecules and the easily accessible and cost-effective OBPs, an OBP-based biosensor was developed for the detection of bitter molecules. Through molecular docking, the amino acid residue-binding sites of the OBP were predicted and analyzed, revealing binding affinities between the OBP and bitter taste molecules, which were used for the evaluation of the interactions between them. Based on the modification of nanomaterials, the interfacial charge-transfer ability of the screen-printed electrodes was improved and the specific surface area was increased for electrochemical impedance spectroscopy measurements. With the detection of bitter substances of different molecular structures, the OBP-based biosensor provided a promising platform for obtaining gustatory information in a fast and simple manner, which could be applied in the field of bitter taste evaluation.

\section{ASSOCIATED CONTENT}

\section{SI Supporting Information}

The Supporting Information is available free of charge at https://pubs.acs.org/doi/10.1021/acsomega.0c04089.

Molecular docking postures of ethanol, denatonium, quinine, and berberine; cyclic voltammograms of screenprinted electrodes under different scan rates; peak currents variations with scan rates; cyclic voltammograms for each modification step of the screen-printed electrodes; SEM images of the screen-printed electrodes; affinity energies between OBPs and ethanol, denatonium, quinine, and berberine; and details of energy scores (PDF)

\section{AUTHOR INFORMATION}

\section{Corresponding Author}

Yanli Lu - Biosensor National Special Laboratory, Key Laboratory for Biomedical Engineering of Education Ministry, Department of Biomedical Engineering, Zhejiang University, Hangzhou 310027, P. R. China; Collaborative Innovation Center of TCM Health Management, Fujian University of Traditional Chinese Medicine, Fuzhou 350108, P. R. China; (1) orcid.org/0000-0003-4577-1936; Phone: +86 571 87953796; Email: yanlilu@zju.edu.cn

\section{Authors}

Zetao Chen - Biosensor National Special Laboratory, Key Laboratory for Biomedical Engineering of Education Ministry, Department of Biomedical Engineering, Zhejiang University, Hangzhou 310027, P. R. China

Qingqing Zhang - Biosensor National Special Laboratory, Key Laboratory for Biomedical Engineering of Education Ministry,
Department of Biomedical Engineering, Zhejiang University, Hangzhou 310027, P. R. China

Jianzhen Shan - The First Affiliated Hospital of Zhejiang University School of Medicine, Hangzhou 310003, P. R. China

Qingjun Liu - Biosensor National Special Laboratory, Key Laboratory for Biomedical Engineering of Education Ministry, Department of Biomedical Engineering, Zhejiang University, Hangzhou 310027, P. R. China; Collaborative Innovation Center of TCM Health Management, Fujian University of Traditional Chinese Medicine, Fuzhou 350108, P. R. China; (1) orcid.org/0000-0002-9442-9355

Complete contact information is available at: https://pubs.acs.org/10.1021/acsomega.0c04089

\section{Notes}

The authors declare no competing financial interest.

\section{ACKNOWLEDGMENTS}

This work was supported by the National Natural Science Foundation of China (Grant Nos. 31671007 and 81801793), National Key Research and Development Program of China (Grant Nos. 2018YFC1707701), and the China Postdoctoral Science Foundation (Grant Nos. 2018M630677 and 2019T120518).

\section{REFERENCES}

(1) Li, H.; Wu, C. J.; Tang, X. Y.; Yu, S. J. Insights into the Regulation Effects of Certain Phenolic Acids on 2,3-Dihydro-3,5dihydroxy-6-methyl-4(H)-pyran-4-one Formation in a Microaqueous Glucose-Proline System. J. Agric. Food Chem. 2019, 67, 9050-9059.

(2) Bahia, M. S.; Nissim, I.; Niv, M. Y. Bitterness prediction in-silico: A step towards better drugs. Int. J. Pharm. 2018, 536, 526-529.

(3) Nissim, I.; Dagan-Wiener, A.; Niv, M. Y. The taste of toxicity: A quantitative analysis of bitter and toxic molecules. IUBMB Life 2017, 69, 938-946.

(4) Beauchamp, G. K.; Mennella, J. A. Early flavor learning and its impact on later feeding behavior. J. Pediatr. Gastroenterol. Nutr. 2009, 48, No. S25.

(5) Li, Y.; Liu, Q.; Xu, Y.; Cai, H.; Qin, L.; Wang, L.; Wang, P. The development of taste transduction and taste chip technology. Chin. Sci. Bull. 2005, 50, 1415-1423.

(6) Lu, L.; Hu, X.; Zhu, Z. Biomimetic sensors and biosensors for qualitative and quantitative analyses of five basic tastes. TrAC, Trends Anal. Chem. 2017, 87, 58-70.

(7) von Molitor, E.; Riedel, K.; Hafner, M.; Rudolf, R.; Cesetti, T. Sensing senses: Optical biosensors to study gustation. Sensors 2020, 20, No. 1811

(8) Hirose, N.; Kawabata, Y.; Kawabata, F.; Nishimura, S.; Tabata, S. Bitter taste receptor T2R1 activities were compatible with behavioral sensitivity to bitterness in chickens. Biochem. Biophys. Res. Commun. 2015, 460, 464-468.

(9) Wu, C.; Du, L.; Zou, L.; Huang, L.; Wang, P. A biomimetic bitter receptor-based biosensor with high efficiency immobilization and purification using self-assembled aptamers. Analyst. 2013, 138, 5989-5994.

(10) Lu, Y.; Huang, Y.; Li, S.; Zhang, Q.; Wu, J.; Xiong, Z.; Xiong, L.; Wan, Q.; Liu, Q. Fat taste detection with odorant-binding proteins (OBPs) on screen-printed electrodes modified by reduced graphene oxide. Sens. Actuators, B 2017, 252, 973-982.

(11) Pelosi, P.; Zhu, J.; Knoll, W. Odorant-binding proteins as sensing elements for odour monitoring. Sensors 2018, 18, No. 3248.

(12) Anholt, R. R. H. Chemosensation and Evolution of Drosophila Host Plant Selection. iScience 2020, 23, No. 100799.

(13) Silva, C.; Matamá, T.; Azoia, N. G.; Mansilha, C.; Casal, M.; Cavaco-Paulo, A. Odorant binding proteins: A biotechnological tool for odour control. Appl. Microbiol. Biotechnol. 2014, 98, 3629-3638. 
(14) He, Y.; Wang, K.; Zeng, Y.; Guo, Z.; Zhang, Y.; Wu, Q.; Wang, $S$. Analysis of the antennal transcriptome and odorant-binding protein expression profiles of the parasitoid wasp Encarsia formosa. Genomics 2020, 112, 2291-2301.

(15) Venthur, H.; Zhou, J. J. Odorant receptors and odorant-binding proteins as insect pest control targets: A comparative analysis. Front. Physiol. 2018, 9, No. 69.

(16) Jeong, Y. T.; Shim, J.; Oh, S. R.; Yoon, H. I.; Kim, C. H.; Moon, S. J.; Montell, C. An odorant-binding protein required for suppression of sweet taste by bitter chemicals. Neuron 2013, 79, 725-737.

(17) Swarup, S.; Morozova, T. V.; Sridhar, S.; Nokes, M.; Anholt, R. R. H. Modulation of feeding behavior by odorant-binding proteins in Drosophila melanogaster. Chem. Senses 2014, 39, 125-132.

(18) Sánchez-Gracia, A.; Vieira, F. G.; Rozas, J. Molecular evolution of the major chemosensory gene families in insects. Heredity 2009, 103, 208-216.

(19) Yasukawa, J.; Tomioka, S.; Aigaki, T.; Matsuo, T. Evolution of expression patterns of two odorant-binding protein genes, OBP57d and OBP57e, in Drosophila. Gene 2010, 467, 25-34.

(20) Kim, M. S.; Repp, A.; Smith, D. P. LUSH odorant-binding protein mediates chemosensory responses to alcohols in Drosophila melanogaster. Genetics 1998, 150, 711-721.

(21) French, A. S.; Sellier, M. J.; Agha Moutaz, A.; Guigue, A.; Chabaud, M. A.; Reeb, P. D.; Mitra, A.; Grau, Y.; Soustelle, L.; Marion-Poll, F. É. R. Dual mechanism for bitter avoidance in drosophila. J. Neurosci. 2015, 35, 3990-4004.

(22) Merriam, J.; Ashburner, M.; Hartl, D. L.; Kafatos, F. C. Toward cloning and mapping the genome of Drosophila. Science 1991, 254, 221-225.

(23) Kalidas, S.; Smith, D. P. Novel genomic cDNA hybrids produce effective RNA interference in adult Drosophila. Neuron. 2002, 33, $177-184$.

(24) Waterhouse, A.; Bertoni, M.; Bienert, S.; Studer, G.; Tauriello, G.; Gumienny, R.; Heer, F. T.; De Beer, T. A. P.; Rempfer, C.; Bordoli, L.; Lepore, R.; Schwede, T. SWISS-MODEL: Homology modelling of protein structures and complexes. Nucleic Acids Res. 2018, 46, W296-W303.

(25) Morris, G. M.; Ruth, H.; Lindstrom, W.; Sanner, M. F.; Belew, R. K.; Goodsell, D. S.; Olson, A. J. AutoDock4 and AutoDockTools4: Automated docking with selective receptor flexibility. J. Comput. Chem. 2009, 30, 2785-2791.

(26) Ji, D.; Liu, Z.; Liu, L.; Low, S. S.; Lu, Y.; Yu, X.; Zhu, L.; Li, C.; Liu, Q. Smartphone-based integrated voltammetry system for simultaneous detection of ascorbic acid, dopamine, and uric acid with graphene and gold nanoparticles modified screen-printed electrodes. Biosens. Bioelectron. 2018, 119, 55-62.

(27) Scott, K. Gustatory Processing in Drosophila melanogaster. Annu. Rev. Entomol. 2018, 63, 15-30.

(28) Winnig, M.; Bufe, B.; Kratochwil, N. A.; Slack, J. P.; Meyerhof, $\mathrm{W}$. The binding site for neohesperidin dihydrochalcone at the human sweet taste receptor. BMC Struct. Biol. 2007, 7, No. 66.

(29) Galindo, K.; Smith, D. P. A large family of divergent Drosophila odorant-binding proteins expressed in gustatory and olfactory sensilla. Genetics 2001, 159, 1059-1072.

(30) Li, Z.; Wei, Y.; Sun, L.; An, X.; Dhiloo, K. H.; Wang, Q.; Xiao, Y.; Khashaveh, A.; Gu, S.; Zhang, Y. Mouthparts enriched odorant binding protein AfasOBP11 plays a role in the gustatory perception of Adelphocoris fasciaticollis. J. Insect Physiol. 2019, 117, No. 103915.

(31) Chu, B.; Chui, V.; Mann, K.; Gordon, M. D. Presynaptic gain control drives sweet and bitter taste integration in Drosophila. Curr. Biol. 2014, 24, 1978-1984.

(32) Beauchamp, G. K. Why do we like sweet taste: A bitter tale? Physiol. Behav. 2016, 164, 432-437.

(33) Lvovskaya, S.; Smith, D. P. A spoonful of bitter helps the sugar response go down. Neuron 2013, 79, 612-614.

(34) Chen, J.; Wang, F. L.; Gui, L. Y.; Zhang, G. H. Identification and tissue distribution of odorant binding protein genes in the citrus fruit fly, Bactrocera minax (Enderlein) (Diptera: Tephritidae). J. AsiaPac. Entomol. 2019, 22, 256-262.
(35) Benedetti, E.; Gavuzzo, E.; Santini, A.; Kent, D. R.; Zhu, Y.-F.; Zhu, Q.; Mahr, C.; Goodman, M. Sweet and bitter taste: Structure and conformations of two aspartame dipeptide analogues. J. Pept. Sci. 1995, 1, 349-359.

(36) Thireou, T.; Kythreoti, G.; Tsitsanou, K. E.; Koussis, K.; Drakou, C. E.; Kinnersley, J.; Kröber, T.; Guerin, P. M.; Zhou, J. J.; Iatrou, K.; Eliopoulos, E.; Zographos, S. E. Identification of novel bioinspired synthetic mosquito repellents by combined ligand-based screening and OBP-structure-based molecular docking. Insect Biochem. Mol. Biol. 2018, 98, 48-61.

(37) Stohs, S. J. Safety, Efficacy, and Mechanistic Studies Regarding Citrus aurantium (Bitter Orange) Extract and p-Synephrine. Phytother. Res. 2017, 31, 1463-1474.

(38) Itoigawa, A.; Hayakawa, T.; Suzuki-Hashido, N.; Imai, H. A natural point mutation in the bitter taste receptor TAS2R16 causes inverse agonism of arbutin in lemur gustation. Proc. R. Soc. B 2019, 286, No. 20190884.

(39) Zhang, Z.; Elfalleh, W.; He, S.; Tang, M.; Zhao, J.; Wu, Z.; Wang, J.; Sun, H. Heating and cysteine effect on physicochemical and flavor properties of soybean peptide Maillard reaction products. Int. J. Biol. Macromol. 2018, 120, 2137-2146.

(40) Di Pizio, A.; Shy, N.; Behrens, M.; Meyerhof, W.; Niv, M. Y. Molecular features underlying selectivity in chicken bitter taste receptors. Front. Mol. Biosci. 2018, 5, No. 874.

(41) Cosconati, S.; Forli, S.; Perryman, A. L.; Harris, R.; Goodsell, D. S.; Olson, A. J. Virtual screening with AutoDock: Theory and practice. Expert Opin. Drug Discovery 2010, 5, 597-607.

(42) Ping, J.; Wang, Y.; Fan, K.; Wu, J.; Ying, Y. Direct electrochemical reduction of graphene oxide on ionic liquid doped screen-printed electrode and its electrochemical biosensing application. Biosens. Bioelectron. 2011, 28, 204-209.

(43) Hutter, E.; Maysinger, D. Gold-nanoparticle-based biosensors for detection of enzyme activity. Trends Pharmacol. Sci. 2013, 34, 497-507.

(44) Pedrosa, V. A.; Caetano, J.; Machado, S. A. S.; Freire, R. S.; Bertotti, M. Acetylcholinesterase immobilization on 3-mercaptopropionic acid self assembled monolayer for determination of pesticides. Electroanalysis 2007, 19, 1415-1420.

(45) Qin, Z.; Zhang, B.; Hu, L.; Zhuang, L.; Hu, N.; Wang, P. A novel bioelectronic tongue in vivo for highly sensitive bitterness detection with brain-machine interface. Biosens. Bioelectron. 2016, 78, 374-380.

(46) Wei, L.; Qiao, L.; Pang, G.; Xie, J. A kinetic study of bitter taste receptor sensing using immobilized porcine taste bud tissues. Biosens. Bioelectron 2017, 92, 74-80.

(47) Liu, Q.; Zhang, D.; Zhang, F.; Zhao, Y.; Jimmy Hsia, K.; Wang, $\mathrm{P}$. Biosensor recording of extracellular potentials in the taste epithelium for bitter detection. Sens. Actuators, B 2013, 176, 497-504.

(48) Wu, C.; Du, L.; Mao, L.; Wang, P. A novel bitter detection biosensor based on light addressable potentiometric sensor. J. Innov. Opt. Health Sci. 2012, 5, No. 1250008.

(49) Hui, G. H.; Ji, P.; Mi, S. S.; Deng, S. P. Electrochemical impedance spectrum frequency optimization of bitter taste cell-based sensors. Biosens. Bioelectron. 2013, 47, 164-170.

(50) Hayashi, N.; Chen, R.; Hiraoka, M.; Ujihara, T.; Ikezaki, H. $\beta$ cyclodextrin/surface plasmon resonance detection system for sensing bitter-Astringent taste intensity of green tea catechins. J. Agric. Food Chem. 2010, 58, 8351-8356.

(51) Saraf, N.; Barkam, S.; Peppler, M.; Metke, A.; VázquezGuardado, A.; Singh, S.; Emile, C.; Bico, A.; Rodas, C.; Seal, S. Microsensor for limonin detection: An indicator of citrus greening disease. Sens. Actuators, B 2019, 283, 724-730.

(52) Cancello, R.; Micheletto, G.; Meta, D.; Lavagno, R.; Bevilacqua, E.; Panizzo, V.; Invitti, C. Expanding the role of bitter taste receptor in extra oral tissues: TAS2R 38 is expressed in human adipocytes. Adipocyte 2020, 9, 7-15. 